\title{
Dosimetric impact of different bladder and rectum filling during prostate cancer radiotherapy
}

\author{
Zhi Chen ${ }^{\dagger}$, Zhaozhi Yang ${ }^{\dagger}$, Jiazhou Wang and Weigang $\mathrm{Hu}^{*}$
}

\begin{abstract}
Background: The aim of this study was to analyze the influence of volumetric changes of bladder and rectum filling on the 3D dose distribution in prostate cancer radiotherapy.

Methods: A total of 314 cone-beam CT (CBCT) image data sets from 19 patients were enrolled in this study. For each $C B C T$, the bladder and rectum were contoured and volume sizes were normalized to those on their original $C T$. The daily delivered dose was recalculated on the CBCT images and the doses to bladder and rectum were investigated. Linear regression analysis was performed to identify the mean dose change of the volume change using SPSS 19.

Results: The data show that the variances of the normalized volume of the bladder and the rectum are $0.13-0.58$ and $0.12-0.50$ respectively. The variances of $V_{70 G y}, V_{60 G y}, V_{50 G y}, V_{40 G y}$ and $V_{30 G y}$ of bladder are bigger than those of rectum for 17 patients. The linear regression analysis indicates a $10 \%$ increase in bladder volume will cause a $5.6 \%$ $( \pm 4.9 \%)$ reduction in mean dose $(p<0.05)$.

Conclusions: The bladder's volume change is more significant than that of the rectum for the prostate cancer patient. The rectum volume variations are not significant except for air bubbles, which change the shape and the position of the rectum. The bladder volume variations may cause dose changes proportionately. Monitoring the bladder's volume before fractional treatment delivery will be crucial for accurate dose delivery.
\end{abstract}

Keywords: Cone-beam CT, Dose tracking, Prostate cancer

\section{Background}

Most radiotherapy patients with prostate cancer are treated with intensity modulated radiotherapy (IMRT). Especially volumetric modulated arc therapy (VMAT) helps to get highly conformal dose distributions for the planning target volume (PTV) while minimizing the dose given to the organs at risk and with short delivery time. However, the advantages of IMRT and VMAT are limited by daily treatment uncertainties [1-3]. Daily treatment uncertainties include daily patient setup errors and internal organ motion and deformation. Studies on prostate motion have shown that both the position and

\footnotetext{
* Correspondence: jackhuwg@gmail.com

${ }^{\dagger}$ Equal contributors

Department of Radiation Oncology, Fudan University Shanghai Cancer Center, 270 DongAn Road, Shanghai 200032, China
}

the shape of the prostate vary throughout the course of treatment $[4,5]$.

Currently, CBCT is a popular imaging method that provides valuable $3 \mathrm{D}$ information of the patient in treatment position [6-8]. In our hospital, we commissioned an Elekta Synergy VMAT accelerator with on-board kVCBCT in 2011, which was used to improve the geometric accuracy of target localization in radiation therapy. In principle, CBCT can be used for dose calculation. Richter et al. reported small dose differences between planning CT and CBCT with $0.9 \% \pm 0.9 \%$ for pelvis, $1.8 \% \pm 1.6 \%$ for thorax and $1.5 \% \pm 2.5 \%$ for head [9]. In our institution, $\mathrm{Hu}$ et al. showed the feasibility of using a region-of-interest (ROI) mapping method for accurate CBCT-based dose calculation [10].

Both rectum and bladder volumes change during the course of radiotherapy for prostate cancer. Roeske et al. 
observed that bladder and rectal volumes varied by $\pm 30 \%$ according to treatment planning computed tomography [11]. Huang et al. used CBCT to analyze the changes of the bladder and rectum during fractionated radiotherapy of the prostate. The differences in the volume and radiation dose were $44 \%( \pm 41)$ and $18 \%( \pm 17)$ for the bladder and $36 \%( \pm 29)$ and $22 \%( \pm 15)$ for the rectum [12]. During the course of image-guided radiotherapy in the post-prostatectomy, Akin et al. assessed the dose-volume parameters $\left(\mathrm{V}_{40 \%}, \mathrm{~V}_{50 \%}, \mathrm{~V}_{60 \%}\right.$, $\mathrm{V}_{65 \%}$ ) for the bladder and rectum, and found the most actual doses delivered were higher than calculated in the treatment planning CT: the $\mathrm{V}_{40 \%}, \mathrm{~V}_{50 \%}, \mathrm{~V}_{60 \%}$ and $\mathrm{V}_{65 \%}$ of the bladder were $20 \%$ more higher than calculated in the treatment planning $\mathrm{CT}$, the $\mathrm{V}_{40 \%}$ of rectum is $8.7 \%$ higher than the calculated in the treatment planning CT, and the $\mathrm{V}_{60 \%}$ of rectum is $59.6 \%$ higher than calculated in the treatment planning $\mathrm{CT}$, while the $\mathrm{V}_{50 \%}$ and $\mathrm{V}_{60 \%}$ of rectum did not show significant difference [13].

The purpose of this study is to track the volume and dosimetric changes in the bladder and rectum based on daily cone-beam CT for prostate radiotherapy. We hope to predict the bladder dose using the volume obtained before treatment.

\section{Methods}

\section{Patient data acquisition}

Nineteen prostate cancer patients were included in this study. Of these, 16 patients with stage T1-3 N0M0 had radiotherapy (RT) with total prescribed dose from 70.2 to 79.2 Gy by 1.8 Gy per fraction. Three patients with biochemical failure after prostatectomy had salvage RT with a total prescribed dose of 64.8 Gy by 1.8 Gy per fraction. For patients with radical RT, the clinical target volume (CTV) was defined as the prostate gland and seminal vesicle as visible on the CT scan. For patients with salvage RT, the CTV included the prostate and seminal vesicle bed. The planned target volume (PTV) was defined as CTV plus $5 \mathrm{~mm}$ in the posterior direction and $8 \mathrm{~mm}$ for all other directions. The rectum, bladder, femoral heads were also contoured for each patient. The patients were required to drink a glass of water about 30 mins before the acquisition of treatment planning CT under the supervision of physicians. Also, the patients were reminded to drink the same amount of water 30 mins before each fractional treatment. The VMAT plan optimization and dose calculation was carried out in the treatment planning system Pinnacle3 (Philips Pinnacle3 V8.0d, Fitchburg, WI, USA). The imaging protocol consisted of a planning CT (AcQsim CT Simulator, Philips Medical System, Cleveland, $\mathrm{OH}$ ) and daily CBCTs (Elekta, Synergy S, XVI, Crawley, UK) acquired in treatment position. The following parameters of $\mathrm{CT}$ and $\mathrm{CBCT}$ were used for imaging: CT: $120 \mathrm{kVp}$, high-resolution reconstruction $(512 * 512)$, with a slice thickness of $5 \mathrm{~mm}$; CBCT: $120 \mathrm{kVp}$, FilterType 'M', reconstruction resolution $(410 * 410)$, with a slice thickness of $3 \mathrm{~mm}$. Each patient's CBCT database contained 8-27 CBCTs. Most of the patient's CBCTs were acquired once every 2 days or twice a week or twice in 3 days. One patient only had the first ten $\mathrm{CBCTs}$ during the treatment course. A total of $314 \mathrm{CBCTs}$ are included in this study. The same physician delineated the bladders and rectums for consistency.

\section{Dose calculation on $\mathrm{CBCT}$}

To calculate the "dose of the day" and assess the dose to the bladder and rectum, the CBCTs were rigidly autoaligned to the planning CT using RayStation treatment planning system (Version 3.0, RaySearch Laboratories AB, Stockholm, Sweden). All the original planning data including the CT data sets, RT structures, and RT plans were exported to the RayStation treatment planning system for dose recalculation. The same Synergy machine was commissioned in the RayStation and the recalculated dose distributions between the Pinnacle and RayStation were verified within $1.5 \%$. Due to the recalculations of the dose on CBCT were performed in the RayStation, the original dose distributions on CT were also recalculated in RayStation which used as plan dose, and comparisons were performed on the same TPS for reliability purpose. In order to use $\mathrm{CBCT}$ images for dose calculation, the image pixel values were converted from $\mathrm{CBCT}$ numbers to physical density using the ROI CT number mapping method as reported [10]. A brief description of the ROI CT number mapping method is as follows: 1) The CBCT images and the CT images of the same patient were acquired on the same day to minimize the change in patient anatomy between the two images. 2) The CT and CBCT images were registered in the RayStation TPS. 3) The ROIs were mapped from $\mathrm{CT}$ to the $\mathrm{CBCT}$, and the mean $\mathrm{CBCT}$ number values of the ROIs were recorded. 4) Generate the $\mathrm{CBCT}$ numbers to physical density calibration curve based on the density values measured on the CT. After that, the original plan was rigidly copied to the $\mathrm{CBCT}$ image data set for dose recalculation.

\section{Dose and volume evaluation}

All bladder and rectum volumes on the CBCTs were normalized to those on the original $\mathrm{CT}$ to track the volume changes. Additionally, the bladder and the rectum were mapped to the planning CT using MIM software version 6.1(MIM Software Inc., Cleveland, OH, USA) to visualize the deformation of the bladder and rectum. The $V_{70 G y}$, $\mathrm{V}_{60 G y}, \mathrm{~V}_{50 G \mathrm{G}}, \mathrm{V}_{40 \mathrm{~Gy}}, \mathrm{~V}_{30 \mathrm{~Gy}}, \mathrm{~V}_{20 \mathrm{~Gy}}$ and $\mathrm{V}_{10 \mathrm{~Gy}}$ for the rectum, and $V_{70 G y}, V_{60 G y}, V_{50 G y}, V_{40 G y}$ and $V_{30 G y}$ for the bladder were calculated respectively. The deviation of the mean 
dose to the bladder and rectum were also evaluated. A linear regression analysis was performed to identify the mean dose change vs. the volume change using SPSS version 19.0 (SPSS, Inc., Chicago, IL, USA). The mean doses and volumes were all normalized to their corresponding dose and volume on the original plan.

\section{Results}

\section{Volume evaluation}

For each patient, the volumes of bladder and rectum on the CBCT were different to those on the planning CT. The deformation of the bladder and the rectum could be clearly identified. Figure 1(a)-(b) show bladder and rectum contours for one patient derived from the different CBCTs projected on one DRR. The corresponding DVHs are displayed as well. Figure 1(c) demonstrates different projections of the bladder contours on one DRR for a patient who had only very slight changed in his bladder filling. The result from the nineteen patients show that the variance of the normalized volume (normalized to corresponding OARs in planning CT) of the bladder is $0.13-0.58$ and the rectum is $0.12-0.50$. Figure 2 shows the variance of the bladder and the rectum for all the patients. Among the 19 patients, 13
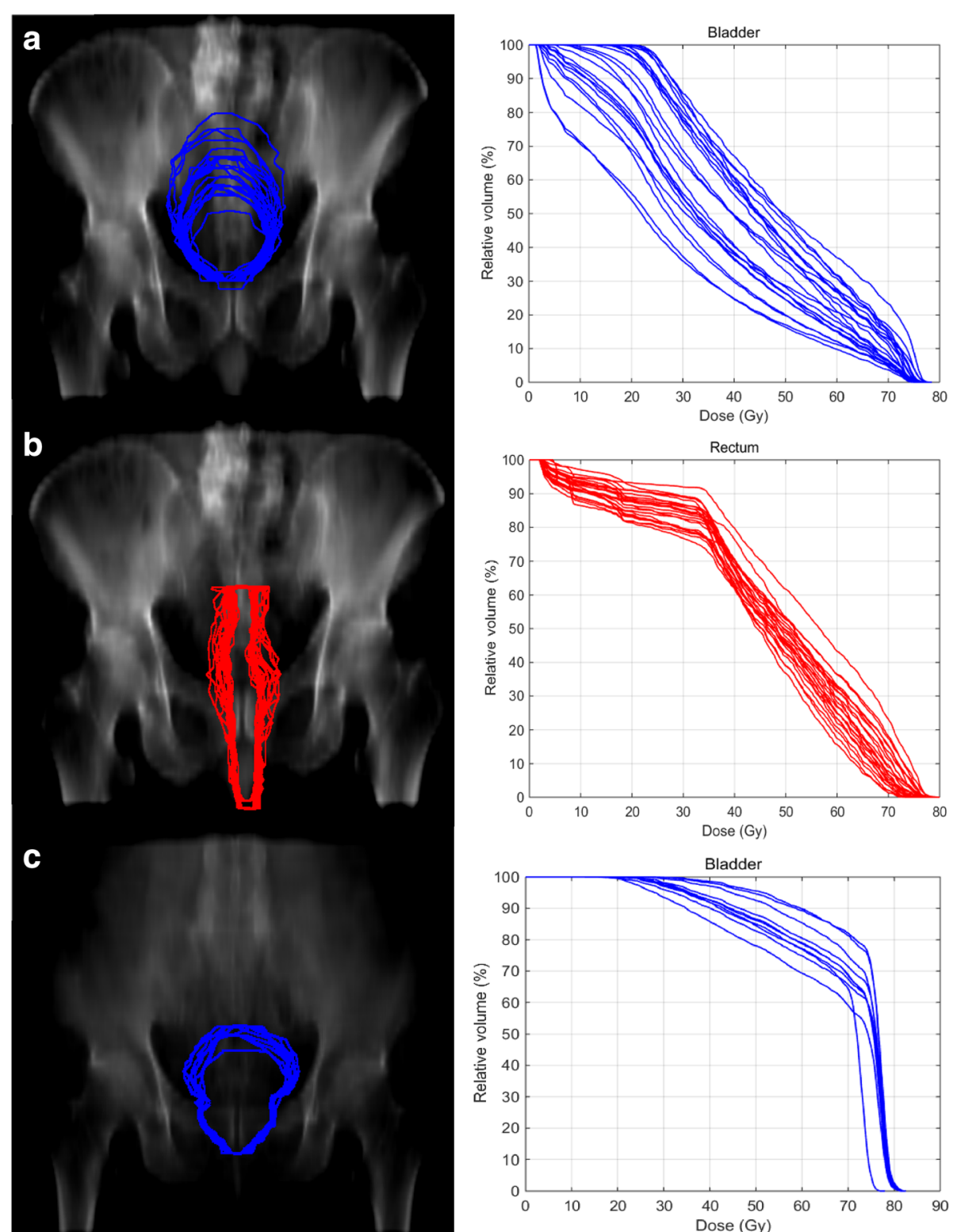

Fig. 1 DRR of two patients. a-b show bladder and rectum contours for one patient derived from the different CBCTs projected on one DRR. The corresponded DVHs are displayed as well. c demonstrates different projections of the bladder contours on one DRR for a patient who had only very slight changed in his bladder filling 

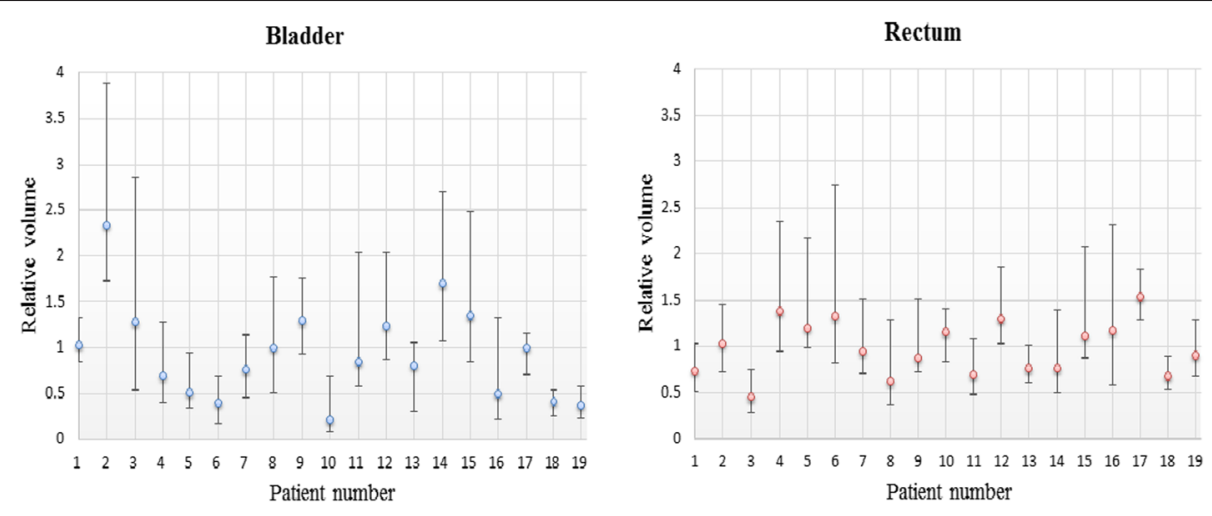

Fig. 2 The variation of the relative volumes for the bladder and rectum

patients' variances of volumes for the rectum are smaller than those of the bladder. But in terms of absolute volume, the variances of the rectum are all smaller than the bladder.

\section{Dose evaluation}

Figure 3(a)-(b) represent dose distribution of the same transversal plane of the same patient in one of CBCT and $\mathrm{CT}$, respectively. Figure 3(d) displays the dose difference between Fig. 3(a) and (b). The dose difference area can be seen on Fig. 3(d) because of the air bubbles which appeared on the CBCT images. The major dose differences which occur around the patient surface are mainly caused by position error and deformation that occurred between fractional $\mathrm{CBCT}$ and planning $\mathrm{CT}$. Because the volume of the rectum varies less than the bladder, as shown in Fig. 4, the results from the 19 patients demonstrate that the standard deviation of $\mathrm{V}_{70 \mathrm{~Gy}}, \mathrm{~V}_{60 \mathrm{~Gy}}, \mathrm{~V}_{50 \mathrm{~Gy}}$, $\mathrm{V}_{40 \mathrm{~Gy}}$ and $\mathrm{V}_{30 \mathrm{~Gy}}$ of the bladders are bigger than those of the rectum for all of the patients except for two. In six of the 19 patients, mean $\mathrm{V}_{\text {70Gy }}$ of the CBCTs are $>20 \%$.

\section{Dose and volume relationship}

Figure 5 displays the regression analysis between the normalized mean dose and the normalized volume for each patient's bladder. The result of the linear regression analysis shows that the mean dose of the bladder would increase while the volume of the bladder shrinks (all with $p<0.05$ ), but find no correlation for the rectum (seven patients $p<0.05$, five of them show positive correlation). On average, the slope of the linear relationship between the mean dose and the volume of the bladder is

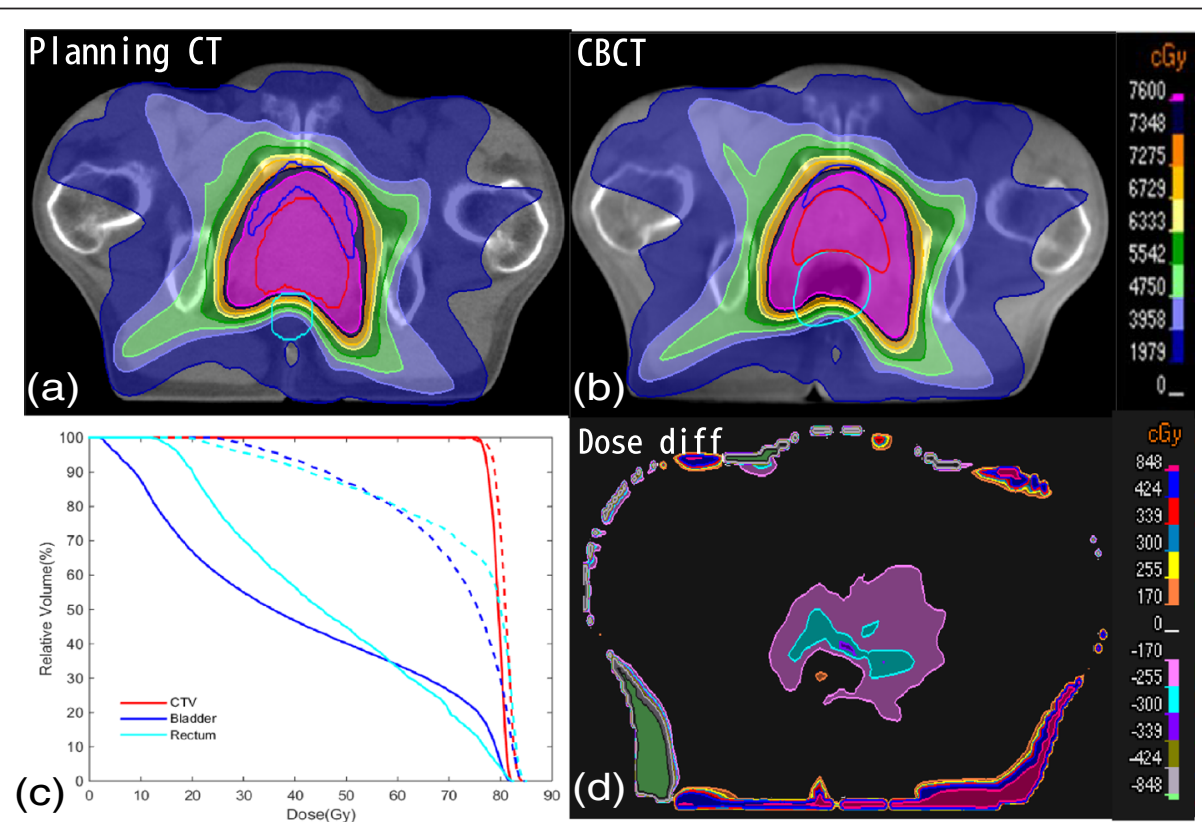

Fig. 3 a-b represent dose distribution of the same transversal plane of the same patient in one of CBCT and CT respectively. $\mathbf{c}$ shows the DVH of the (a) (solid line) and (b) (dash line). $\mathbf{d}$ displays the dose difference between the (a) and (b) 


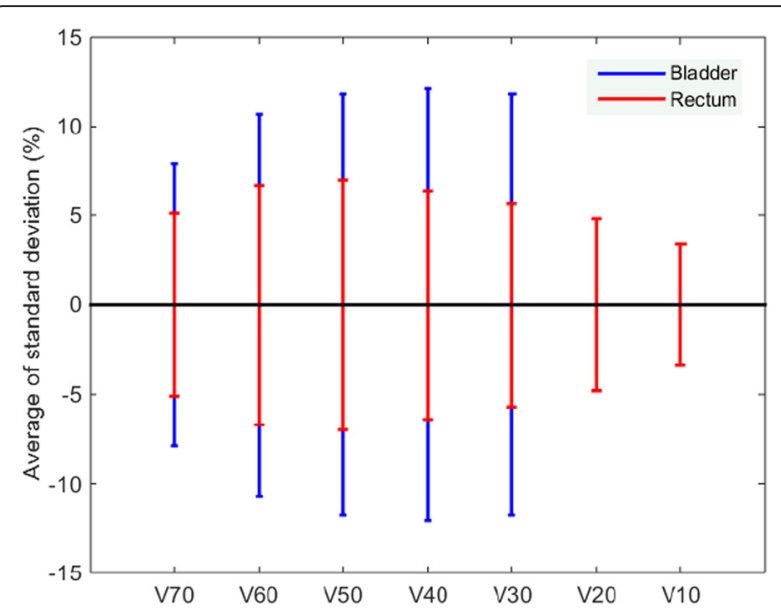

Fig. 4 The average of standard deviation of the $V_{70 G y}, V_{60 G y}, V_{50 G y}$ $V_{40 G y}$ and $V_{30 G y}$ of the bladders and the $V_{70 G y}, V_{60 G y}, V_{50 G y}, V_{40 G y}$ $V_{30 G y}, V_{20 G y}$ and $V_{10 G y}$ of the rectum for the all 19 patients

$0.56 \pm 0.49$, which means if the bladder volume is increased by $10 \%$, the mean dose will reduce by $5.6 \%$ $( \pm 4.9 \%)$.

\section{Discussion}

Previous works showed that the position and shape of the bladder and rectum vary throughout the course of prostate radiotherapy treatment [14-16]. The application of $\mathrm{CBCT}$ can improve the accuracy of radiation therapy in target location and dose delivered $[17,18]$. In this study, we used $\mathrm{CBCT}$ for patient dose recalculation and organs at risk evaluation. Although patient setup error can be corrected through the rigid registration, deformations like the air bubbles in the rectum and the volume variance of the bladder appear to be more important. Fortunately, these deformations only affect the dose distribution slightly in the central area of the patient. The maximum dose difference between initial treatment plan and recalculated dose for the $\mathrm{CBCT}$ in the central area is no more than $3.50 \mathrm{~Gy}$, about 5.1$4.2 \%$ of the prescription dose $(64.8-79.2$ Gy) to the PTV. Therefore, the CTV dose coverage would be sufficient if the CTV does not move out of the PTV area (projected from the original planning CT). It's necessary to start to monitor the rectum and bladder filling at the treatment planning $\mathrm{CT}$. If rectum and bladder filling are not sufficient, the treatment delivery should be rejected.

The most significant variation in dose area is caused by the air bubbles, which expands the rectum closer to the target. This is one major reason for variations which appeared in rectum dose-volume value. Moreover, the image quality of $\mathrm{CBCT}$ suffers from the air bubbles, which blurs the boundary of the rectum and causes uncertainty both on contouring and dose evaluation. Padhani et al. had subjectively graded 5 levels of rectal air/ fecal distension and found the moderate and large grades, similar to the cases with air bubbles in this study, were the most likely to produce rectal movements [19]. He suggested that patients should be advised to empty their rectum prior to radiotherapy to reduce rectal distension. To increase the accuracy of the dose distribution, the patients should empty the rectum before radiotherapy treatments.

Bladder shrinkage and expansion are two key factors for dose variances, but are hard to control, especially in cancer centers without careful bladder filling exam. This study found that most patients' bladder volume varied during treatment. What's more, bladder volumes of three patients were all smaller than their original state, while in one patient the opposite case was true. Perhaps some patients may not feel comfortable holding urine and did not realize the importance of drinking water to keep the bladder full, so they did not always drink water. Furthermore, some patients may be too nervous and therefore drank more water, making the bladder full. In our hospital, there are too many patients that need to be treated, so physicians and technicians may not always be able to relax the patient or confirm whether the patient drink water before treatment. Patients could not keep the bladder volume constant during each fraction treatment while the air bubbles only existed in a few CBCTs during the treatment course, which might result in the volume of the bladder vary more than the rectum in this study. An increase of $10 \%$ of bladder volume will cause to a $5.6 \%$ reduction of mean dose. This indicates that some new method with quick bladder volume evaluation will be useful for ensuring the accurate dose delivery and sparing the bladder. As Fig. 3 shows, the shape of the rectum can experience irregular changes because of the air bubbles which can decrease the chances of finding a statistical association between the rectum volume and the mean dose.

There are several studies which have sought to define normal tissue dose constraints based on retrospective analyses of dose-volume and toxicity relationships. Pederson et al. found that the rate of Grade 2+ gastrointestinal (GI) toxicity were correlated to $\mathrm{V}_{70 \mathrm{~Gy}}, \mathrm{~V}_{65 \mathrm{~Gy}}$ and $\mathrm{V}_{40 \text { Gy }}$ of the rectum. Freedom from Grade 2+ GI toxicity at 4 years was $100 \%$ for men with rectal $V_{70 G y}<10 \%$, $\mathrm{V}_{65 \mathrm{~Gy}}<20 \%$, and $\mathrm{V}_{40 \mathrm{~Gy}}<40 \%$; $92 \%$ for men with rectal $\mathrm{V}_{70 \mathrm{~Gy}}<20 \%, \mathrm{~V}_{65 \mathrm{~Gy}}<40 \%$, and $\mathrm{V}_{40 \mathrm{~Gy}}<80 \%$; and $85 \%$ for men exceeding these criteria [20]. Another study showed that the risk of chronic rectal toxicity Grade $>=$ 2 for rectal with $\mathrm{V}_{70 \mathrm{~Gy}}<15 \%$ was $9 \%, \mathrm{~V}_{70 \mathrm{~Gy}}$ between 25 and $40 \%$ was $18 \%$ and $V_{70 G y}>40 \%$ was $25 \%$ [21]. They all pointed out that the higher the rectal volume receiving high doses ( $>40 \mathrm{~Gy}$ ), especially $70 \mathrm{~Gy}$, the higher the probability that the patient would get Grade 2+ GI. This suggests that when using $\mathrm{CBCT}$ to improve the accuracy 

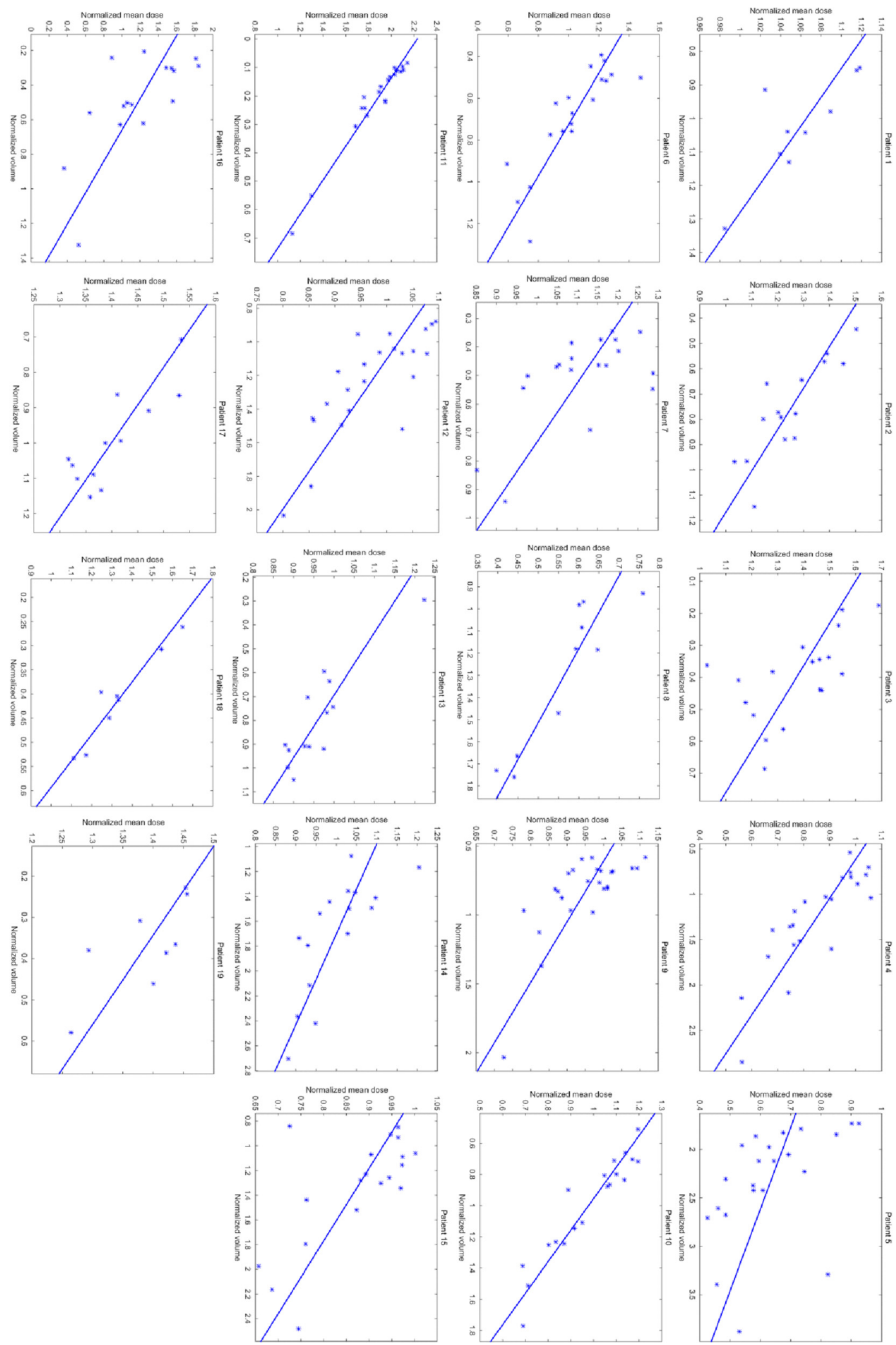

Fig. 5 The regression analysis between the normalized mean dose and the normalized volume for each patient's bladder 
of the target location, we should also consider keeping the rectum from the high dose area in order to reduce the risk of Grade 2+ GI. As for the bladder, only a few studies found dose-volume relationships associated with the risk of GU toxicity [22]. Perhaps the changes in bladder filling cause changes in bladder DVH parameters which make the establishment of a relationship between the dose-volume and GU toxicity difficult. But it is still important to reduce the volume of bladder which enters the high dose area when using the CBCT to set the patient's position.

In this study, we only focused on dose verification of the bladder and the rectum for those treated patients. Our results suggest that the current patient instruction with only drink a glass of water is not sufficient to ensure the bladder filling in a busy center. Pretreatment CBCT imaging or quick bladder volume evaluation tool is required for accurate treatment delivery. Especially for the quick bladder volume evaluation tool without additional imaging dose and patient setup time will be a good option for busy center.

\section{Conclusion}

This study used daily cone-beam CT to evaluate the volume changes of bladder and rectum and evaluate the dosimetric changes of bladder and rectum. Our results demonstrate that the volume change of the bladder is more significant than changes in rectal volume for prostate cancer patients. The volume changes of the rectum are not significant except for the air bubbles in the rectum, but bladder volume variations will cause bladder dose changes proportionately. The mean dose received to the bladder will decrease with enlarging the volume. In addition, the CTV will still be covered by the isodose of $95 \%$ of prescription dose during the treatment course.

\section{Abbreviations}

CBCT, cone-beam CT; CTV, clinical target volume; IMRT, intensity modulated radiotherapy; PTV, planned target volume; ROI, region-of-interest;

$\mathrm{RT}$, radiotherapy; VMAT, volumetric modulated arc therapy

\section{Acknowledgements}

The authors thank Joshua Ye for helpful discussion and editing of the paper.

\section{Funding}

This study is supported by the National Natural Science Foundation (11205033).

Availability of data and materials

The original data will not be shared according to the department rules.

\section{Authors' contributions}

ZY carried out patient treatment. All authors carried out the study and conceived of the study. ZC and WH draft the manuscript.

All authors read and approved the final manuscript.

\section{Competing interests}

The authors declare that they have no competing interests.

Consent for publication

Not applicable.
Ethics approval and consent to participate

Not applicable.

Received: 2 September 2015 Accepted: 28 July 2016

Published online: 02 August 2016

\section{References}

1. Alasti H, Petric MP, Catton CN, Warde PR. Portal imaging for evaluation of daily on-line setup errors and off-line organ motion during conformal irradiation of carcinoma of the prostate. Int J Radiat Oncol. 2001;49(3):869-84.

2. Byrne TE. A review of prostate motion with considerations for the treatment of prostate cancer. Med Dosim. 2005;30(3):155-61.

3. Kupelian PA, Langen KM, Zeidan OA, Meeks SL, Willoughby TR, Wagner TH, Jeswani S, Ruchala KJ, Haimerl J, Olivera GH. Daily variations in delivered doses in patients treated with radiotherapy for localized prostate cancer. Int J Radiat Oncol. 2006;66(3):876-82.

4. Britton KR, Takai Y, Mitsuya M, Nemoto K, Ogawa Y, Yamada S. Evaluation of inter- and intrafraction organ motion during intensity modulated radiation therapy (IMRT) for localized prostate cancer measured by a newly developed on-board image-guided system. Radiat Med. 2005;23(1):14-24.

5. Huang E, Dong L, Chandra A, Kuban DA, Rosen II, Pollack A. Intrafraction prostate motion during IMRT for prostate cancer. Int J Radiat Oncol. 2002;53(2):261-8.

6. Kim GY, Pawlicki T, Le QT, Luxton G. Linac-based on-board imaging feasibility and the dosimetric consequences of head roll in head-and-neck IMRT plans. Med Dosim. 2008;33(1):93-9.

7. Nill S, Seeber A, Hesse B, Huber P, Thilmann C. Linac-integrated kV-cone beam CT: technical features and first applications. Med Dosim. 2006;31(1):62-70.

8. Gillis A, Chen J, Aubin M, Bucci MK. Megavoltage cone-beam CT: system description and clinical applications. Med Dosim. 2006;31(1):51-61.

9. Richter A, Hu Q, Steglich D, Baier K, Wilbert J, Guckenberger M, Flentje M. Investigation of the usability of conebeam CT data sets for dose calculation. Radiat Oncol. 2008;3(6):7215-23.

10. Hu W, Ye J, Wang J, Ma X, Zhen Z. Use of kilovoltage X-ray volume imaging in patient dose calculation for head-and-neck and partial brain radiation therapy. Radiat Oncol. 2010;5(12):29.

11. Roeske JC, Forman JD, Mesina CF, He T, Pelizzari CA, Fontenla E, Vijayakumar $\mathrm{S}$, Chen GT. Evaluation of changes in the size and location of the prostate, seminal vesicles, bladder, and rectum during a course of external beam radiation therapy. Int J Radiat Oncol. 1995;33(5):1321-9.

12. Huang TC, Chou KT, Yang SN, Chang CK, Liang JA, Zhang G. Fractionated changes in prostate cancer radiotherapy using cone-beam computed tomography. Med Dosim. 2015;40(3):222-5.

13. Akin M, Öksüz DC, Iktueren B, Ambarcioglu P, Karacam S, Koca S, Dincbas F. Does rectum and bladder dose vary during the course of image-guided radiotherapy in the postprostatectomy setting? Tumori. 2014;100:529-35.

14. Yong Y, Eduard S, Tianfang L, Chuang W, Lei X. Evaluation of on-board kV cone beam $\subset$ (CBCT)-based dose calculation. Phys Med Biol. 2007:52(3):685-705(621).

15. Thilmann C, Nill S, Tucking T, Hesse B, Dietrich L, Rhein B, Haering P, Oelfke $U$, Debus J, Huber P. Correction of patient positioning errors based on inline cone beam CTs: clinical implementation and first experiences. Radiat Oncol. 2005;63(6):550551.

16. Grills IS, Hugo G, Kestin LL, Chao KK, Wloch J, Yan D. Image guided radiotherapy (IGRT) via online cone beam CT substantially reduces margin requirements for stereotactic lung radiotherapy. Int J Radiat Oncol. 2008;70(3):1045-56.

17. Li T, Thongphiew D, Zhu X, Lee WR, Vujaskovic Z, Yin FF, Wu QJ. Adaptive prostate IGRT combining online re-optimization and re-positioning: a feasibility study. Phys Med Biol. 2011;56(5):1243-58.

18. Wu Q, Thongphiew D, Wang Z, Mathayomchan B, Chankong V, Yoo S, Lee W, Yin F. On-line re-optimization of prostate IMRT plans for adaptive radiation therapy. Phys Med Biol. 2008;53(3):673-91.

19. Padhani AR, Khoo VS, Suckling J, Husband JE, Leach MO, Dearnaley DP. Evaluating the effect of rectal distension and rectal movement on prostate gland position using cine MRI. Int J Radiat Oncol. 1999;44(3):525-33.

20. Pederson AW, Fricano J, Correa D, Pelizzari CA, Liauw SL. Late toxicity after intensity-modulated radiation therapy for localized prostate cancer: an exploration of dose-volume histogram parameters to limit genitourinary and gastrointestinal toxicity. Int J Radiat Oncol. 2012;82(1):235-41.

21. Carlos V, Alvaro M, Kestin LL, Di Y, Inga G, Brabbins DS, Lockman DM, Jian L, Gustafson GS, Chen PY. Dose-volume analysis of predictors for chronic 
rectal toxicity after treatment of prostate cancer with adaptive imageguided radiotherapy. Int J Radiat Oncol Biol Phys. 2005;62(5):1297-308.

22. Carillo V, Cozzarini C, Rancati T, Avuzzi B, Botti A, Borca VC, Cattari G, Civardi F, Esposti CD, Franco P. Relationships between bladder dose-volume/surface histograms and acute urinary toxicity after radiotherapy for prostate cancer. Radiother Oncol. 2014;111(1):100-5.

Submit your next manuscript to BioMed Central and we will help you at every step:

- We accept pre-submission inquiries

- Our selector tool helps you to find the most relevant journal

- We provide round the clock customer support

- Convenient online submission

- Thorough peer review

- Inclusion in PubMed and all major indexing services

- Maximum visibility for your research

Submit your manuscript at www.biomedcentral.com/submit 